\title{
Why does necking ignore notches in dynamic tension?
}

\author{
Y. Rotbaum, S. Osovski, and D. Rittel ${ }^{\mathrm{a}}$ \\ Faculty of Mechanical Engineering, Technion, 32000 Haifa, Israel
}

\begin{abstract}
Recent experimental work has revealed that necking of tensile specimens, subjected to dynamic loading, is a deterministic phenomenon, governed by the applied boundary conditions. Furthermore it was shown that the potential sited, dictated by the boundary conditions, may prevail even in the presence of a notch, thus necking may occur away of the notched region. The present paper combines experimental and numerical work to address this issue. Specifically, it is shown that the dynamic tensile failure locus is dictated by both the applied velocity boundary condition and the material mechanical properties, specifically strain-rate sensitivity and strain-rate hardening. It is shown that at sufficiently high impact velocities, the flows stress in the notch vicinity becomes quite higher than in the rest of the specimen, so that while the former resists deformation, it transfers the load to the latter, resulting in the formation of a local neck and failure away from the notch. Small local perturbations in the material properties are shown to be sufficient to stabilize the structure under local failure until a neck forms elsewhere. While the physical observations are quite counterintuitive with respect to the engineering views of stress concentrator's effect, the present work rationalizes those observations and also provides information for the designers of dynamically tensioned structures that may contain notches or similar flaws.
\end{abstract}

\section{Necking}

The mechanical response of a structural element under external loads may be strongly influenced by the presence of geometrical perturbations, amounting to a local increase in the stress field surrounding it. The importance of notched members under tension has led to a vast body of works focusing on evaluating the stress intensity factors around geometrical discontinuities [1-4], and specifically the differences between quasi-static and dynamic loading scenarios [5-7]. An underlying assumption in all of the above-mentioned studies is that the fracture locus will be that of the geometrical imperfection, or any other given flaw, even in situations where inertia plays an important role. Material heterogeneities related to the manufacturing process are vastly ignored or considered secondary, with respect to the presence of the dominant flaw. It was recently shown that under dynamic tensile loading, the necking locations is a deterministic event resulting from the applied boundary conditions [8]. Furthermore, it was shown experimentally that the necking location, as dictated by the applied boundary conditions, may prevail even in the presence of a geometrical perturbation introduced as a notch [9]. The results of presented in [9] imply that the presence of a geometrical imperfection will not necessarily dictate the dynamic failure locus, as commonly assumed in the literature.

Here we present a hybrid experimental-numerical study, examining several potent physical factors responsible for the selection of the dynamic failure locus.

Experimental results for 90 dynamic tensile notched 15-5 PH (annealed) and 4340 steel specimens are presented, and are shown to follow the trends found in [9],

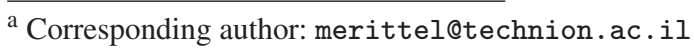

a

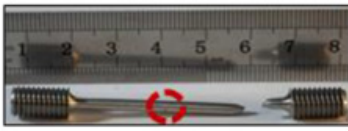

b

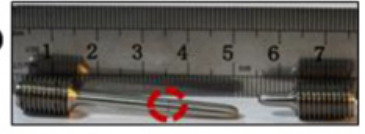

c
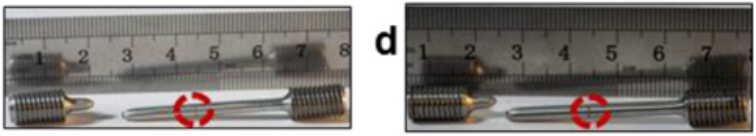

Figure 1. Dynamically loaded notched tensile specimens showing failure outside of the notch. Specimens shown in (a) and (b) were loaded at $15 \mathrm{~m} / \mathrm{s}$ and failed on the impacted side. Specimens shown in (c) and (d) were loaded at $16 \mathrm{~m} / \mathrm{s}$ and $18 \mathrm{~m} / \mathrm{s}$ respectively and failed at the opposite side.

as shown in Fig. 1. The notch depth was chosen as such that will ensure failure within the notch under quasi-static loading.

The dynamic tensile tests were modeled using the commercial finite element package Abaqus explicit [10]. The boundary conditions used in the simulations, were set as a symmetrical trapezoidal velocity profile, applied on the free surface of the incident bar, with maximum amplitude in the range of $7-21 \mathrm{~m} / \mathrm{s}$. The pulse duration was taken to be the same as in the experiments. The response of the elastic viscoplastic material is calculated within the framework of $J_{2}$ flow theory. The viscoplastic response was taken as such that will account for increased rate sensitivity at high strain rates, following Zhou et al. [11]. The viscoplastic response is described by a power law up to a transition strain rate $\dot{\varepsilon}_{m}$ from which it becomes exponentials. For a detailed description of the numerical mode, the reader is referred to [12]. The material properties used in the simulations are of a generic character and do not represent accurately the experimental materials of

This is an Open Access article distributed under the terms of the Creative Commons Attribution License 4.0, which permits unrestricted use, distribution, and reproduction in any medium, provided the original work is properly cited. 


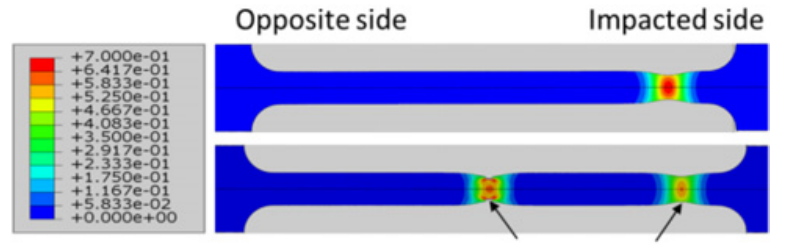

Figure 2. Numerical simulation of dynamically deformed representative steel specimens stretched at a peak velocity of $17.5[\mathrm{~m} / \mathrm{s}]$. The upper specimen is smooth while the lower contains a $10 \%$ notch. The color map represents the equivalent plastic strain values.
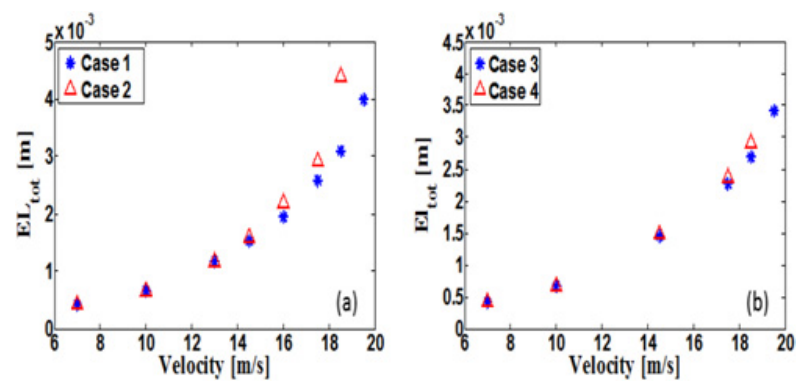

Figure 3. Macroscopic elongation at equal radial displacement values (meeting time). (A) Cases 1 and 2. (b) Cases 3 and 4.

the study. As such, they are used to explore trends and not reproduce experimental results. Representative results of the numerical simulations of smooth and $10 \%$ centernotched samples, stretched at a peak velocity of $17.5 \mathrm{~m} / \mathrm{s}$, are shown in Fig. 2. For a smooth specimen, the failure location is on the impacted side, in correspondence with [8]. For the notched specimen, one observes a strong competition between the neck on the impacted side and the imperfection in the middle of the gauge. This is the outcome of the joint influence of inertia and stress waves against local the stress concentration.

In the following, the competition between potential necking sites is described by comparing the evolution of the local radial displacement with respect to the total elongations. The overall elongation at which the radial displacement in the two potential sites is equal, provides an estimation as to the prevalence of the notched site over the one dictated by the boundary conditions. Once the two curves meet, the displacement under one of them starts to grow rapidly while the other is unloaded. The role of the viscoplastic behavior was studied by varying the transition strain rate $\dot{\varepsilon}_{m}$ from $8000 \mathrm{~s}^{-1}$ (cases $1 \& 2$ ) to from $16000 \mathrm{~s}^{-1}$ (cases $3 \& 4$ ). The exponent of the rate dependency at high strain rate of cases $1 \& 3$ is identical and lower than the one used in cases $2 \& 4$. In Fig. 3 the effect of the viscoplastic behavior on the necking location is presented. One should emphasize that the case with a higher elongation prior to the prevalence of one of the potential sites, is more stable in the sense that larger total elongation to fracture can be reached. Thus, specimens at whom the competition between the two localization sites lasts longer, promotes a higher energy absorption capability.

An important issue, which is generally overlooked, concerns the machining of the notch. The latter

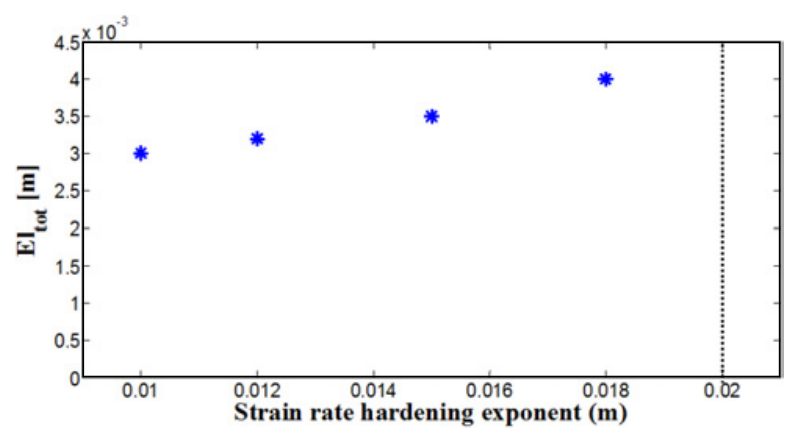

Figure 4. The nominal elongation of the specimen at the meeting time for $\mathrm{V}_{\max }=18.5 \mathrm{~m} / \mathrm{s}$, for different values of $\mathrm{m}_{\text {notch }}$. Note that for $\mathrm{m}=0.02$, there is no meeting point as the neck dominates throughout the process.

has a definite local hardening effect, which can be somewhat mitigated by annealing treatments. An obvious consequence of the machining process is an increase in the yield strength due to the storage of cold work. The effect of small variations in the strain rate sensitivity was studied for case 1 with a fixed maximal velocity of $18.5 \mathrm{~m} / \mathrm{s}$. For that purpose an area with a radius of $130 \mu \mathrm{m}$ around the notch root was selected to have slightly different material properties than the rest of the specimen. The strain rate hardening exponent in the vicinity of the notch-tip was varied in the range $\mathrm{m}_{\text {notch }}=0.012,0.015,0.017,0.020$. The maximum difference in stress between the notch and the rest of the specimen at a plastic strain of $40 \%$ for $\mathrm{m}_{\text {notch }}=0.020$ is $48 \mathrm{MPa}$ which corresponds to $\sim 4.65 \%$. In Fig. 4 the total elongation up to the meeting time of the radial displacements in the two potential sites is plotted with respect to $m_{\text {notch. }}$ As evident from Fig. 4, when the notch area is more hardened, the sample elongates more before reaching to its final failure state, indicating that the notch is less dominant in the competition between the potential sites. For $\mathrm{m}_{\text {notch }}=0.02$, the necking site dictated by the boundary conditions prevailed throughout the deformation process. Therefore one can suggest that minor perturbations in the material flow stress can cause a significant change in the overall structural response.

The main conclusions arising from the work presented here are:

1. The locus of dynamic tensile failure of a flawed (notched) structure is dictated by the applied velocity as a boundary condition, strain rate sensitivity and ratehardening of the material.

2. Small perturbations in the material's constitutive behavior, resulting from the machining process, can promote a significant change in the structural response under dynamic tensile loads.

3. At sufficiently high velocities, the very high strain rates in the notch stabilize the deformation and transfer the load to another part of the specimen which ultimately grows a neck there.

4. When designing a tensile notched structural component, the dynamic mechanical properties and boundary conditions should both be carefully taken into account to predict the failure locus. 


\section{References}

[1] E. Coker, K. Chakko, Y. Satake, Proc Inst Eng Shipbuilding Scotland, 63, 34-94 (1919)

[2] R. Howland, Phil. Trans. Roy. Soc. Series A, Containing Papers of a Mathematical or Physical Character: 49-86 (1930)

[3] M. Strandberg, Eng. Fract. Mech., 68, 577-585 (2001)

[4] M. Zappalorto, P. Lazzarin, Eng. Fract. Mech., 78, 2691-2706 (2011)

[5] W. James, W. North, J Strain Anal, 4, 261-266 (1969)

[6] N. Nakayama, M. Ohashi, H. Takeishi, JSME Int. J Series A, Solid Mech. Mat. Eng, 41, 326-331 (1998)
[7] H. Matsumoto, T. Adachi, Y. Kakuhama, K. Fukuzawa, JSME Int. J. Ser 1, Solid Mech., Strength of Materials, 33, 37-43 (1990)

[8] S. Osovski, D. Rittel, J.A. Rodríguez-Martínez, R. Zaera, Dynamic tensile necking: influence of specimen geometry and boundary conditions. Mech. Mat. 62, 1-13 (2013)

[9] D. Rittel, Y. Rotbaum, J.A. Rodríguez-Martínez, D. Sory, R. Zaera, Exp. Mech., 54(6), 1099-1109 (2014)

[10] Hibbett, Karlsson, Sorensen, Hibbitt. (1998) ABAQUS/standard: User's Manual, Hibbitt, Karlsson \& Sorensen

[11] M. Zhou, A. Needleman, R.J. Clifton, J. Mech. Phys. Sol., 42, 423-458 (1994)

[12] Y. Rotbaum, S. Osovski, D. Rittel, J. Mech. Phys. Sol., 78, 173-185 (2015) 